\title{
85.
}

\section{NOTE ON A FAMILY OF CURVES OF THE FOURTH ORDER.}

[From the Cambridge and Dublin Mathematical Journal, vol. v. (1850), pp. 148-152.]

THE following theorem, in a slightly different and somewhat less general form, is demonstrated in Mr Hearn's "Researches on Curves of the Second Order, \&c.", Lond. 1846: "The locus of the pole of a line, $u+v+w=0$, with respect to the conics passing through the angles of the triangle $(u=0, v=0, w=0)$, and touching a fixed line $\alpha u+\beta v+\gamma w=0$, is the curve of the fourth order,

$$
\sqrt{ }\{\alpha u(v+w-u)\}+\sqrt{ }\{\beta v(w+u-v)\}+\sqrt{ }\{\gamma w(u+v-w)\}=0 ; "
$$

the difference in fact being, that with $\mathrm{Mr}$ Hearn the indeterminate line $u+v+w=0$ is replaced by the line $\infty$, so that the poles in question become the centres of the conics.

Previous to discussing the curve of the fourth order, it will be convenient to notice a property of curves of the fourth order with three double points. Such curves contain eleven arbitrary constants: or if we consider the double points as given, then five arbitrary constants. From each double point may be drawn two tangents to the curve; any five of the points of contact of these tangents determine the curve, and consequently determine the sixth point of contact. The nature of this relation will be subsequently explained; at present it may be remarked that it is such that, if three of the points of contact (each one of such points of contact corresponding to a different double point) lie in a straight line, the remaining three points of contact also lie in a straight line. A curve of the fourth order having three given double points and besides such that the points of contact of the tangents from the double points lie three and three in two straight lines, contains therefore four arbitrary constants. Now it is easily seen that the curve in question has three double points, viz. the points given by the equations

$$
(u=0, v-w=0), \quad(v=0, w-u=0), \quad(w=0, u-v=0),
$$

points which may be geometrically defined as the projections from the angles of the triangle $(u=0, v=0, w=0)$ upon the opposite sides, of the point $(u=v=w)$ which is the harmonic with respect to the triangle of the given line $u+v+w=0$. Moreover, 
the lines $u=0, v=0, w=0$ (being lines which, as we have seen, pass through the double points) touch the curve in three points lying in a line, viz. the given line $\alpha u+\beta v+\gamma w=0$. Hence the curve in question is a curve with three double points, such that the points of contact of the tangents from the double points lie three and three in two straight lines. Considering the double points as given, the functions $u, v, w$ contain two arbitrary ratios, and the ratios of the quantities $\alpha, \beta, \gamma$ being arbitrary, the equation of the curve contains four arbitrary constants, or it represents the general curve of the class to which it has been stated to belong.

As to the investigation of the above-mentioned theorem with respect to curves of the fourth order with three double points, the general form of the equation of such a curve is

$$
\frac{a}{x^{2}}+\frac{b}{y^{2}}+\frac{c}{z^{2}}+\frac{2 f}{y z}+\frac{2 g}{z x}+\frac{2 h}{x y}=0,
$$

where the double points are the angles of the triangle $(x=0, y=0, z=0)$. It may be remarked in passing, that the six tangents at the double point touch the conic

$$
a x^{2}+b y^{2}+c z^{2}-2 f y z-2 g x z-2 h x y=0 .
$$

To determine the tangents through $(y=0, z=0)$, we have only to write the equation to the curve under the form

$$
\left(\frac{a}{x}+\frac{h}{y}+\frac{g}{z}\right)^{2}+\frac{C}{y^{2}}+\frac{B}{z^{2}}-\frac{2 F}{y z}=0:
$$

the points of contact are given by the system

$$
\begin{aligned}
& \frac{a}{x}+\frac{h}{y}+\frac{g}{z}=0 \\
& \frac{C}{y^{2}}+\frac{B}{z^{2}}-\frac{2 F}{y z}=0
\end{aligned}
$$

the latter equation (which evidently belongs to a pair of lines) determining the tangents. The former equation is that of a conic passing through the angles of the triangle $x=0, y=0, z=0$ : since the tangents pass through the point $(y=0, z=0)$ they evidently each intersect the conic in one other point only. The equation of the tangents shows that these lines are the tangents through the point $y=0, z=0$ to the conic whose equation is

$$
a A^{2} x^{2}+b B^{2} y^{2}+c C^{2} z^{2}+2 f B C y z+2 g C A z x+2 h A B x y=0 .
$$

To complete the construction of the points of contact it may be remarked, that the equations which determine the coordinates of these points may be presented under the form

$$
\begin{aligned}
& A x=\{A \\
& B y=\left\{H-g \sqrt{ }\left(\frac{-K}{a}\right)\right\} x, \\
& C z=\left\{G+h \sqrt{ }\left(\frac{-K}{a}\right)\right\} x,
\end{aligned}
$$

C. 
whence also

$$
\begin{array}{lr}
a A x+h B y+g C z= & K x \\
h A x+b B y+f C z= & G \sqrt{\left(\frac{-K}{a}\right) x} \\
g A x+f B y+c C z & =-H \sqrt{ }\left(\frac{-K}{a}\right) x
\end{array}
$$

or writing for shortness $\xi, \eta, \zeta$ instead of the linear functions forming the first sides of these equations respectively, we have

$$
\frac{\xi}{\sqrt{(-a K)}}=\frac{\eta}{G}=-\frac{\zeta}{H}
$$

from which it follows at once that the equation to the line forming the two points of contact is

$$
\frac{\eta}{G}+\frac{\zeta}{H}=0 ;
$$

and this may again be considered as the line joining the points $\left(\xi=0, \frac{\xi}{F}+\frac{\eta}{G}+\frac{\zeta}{H}=0\right)$ and $(\eta=0, \zeta=0)$.

Now $\xi=0, \eta=0, \xi=0$, are the polars of the double points (or angles of the triangle $x=0, y=0, z=0)$ with respect to the last-mentioned conic. The equation of the line joining the point $(y=0, z=0)$ with the point $(\eta=0, \zeta=0)$, is easily seen to be $G B y-C H z=0$; from which it follows, that the lines forming each double point with the intersection of the polars of the other two double points meet in a point, the coordinates of which are given by

$$
x: y: z=\frac{1}{A F}: \frac{1}{B G}: \frac{1}{C H},
$$

and the polar of this point is the line $\frac{\xi}{F}+\frac{\eta}{G}+\frac{\zeta}{H}=0$. The construction of this line is thus determined; and we have already seen how this leads to the construction of

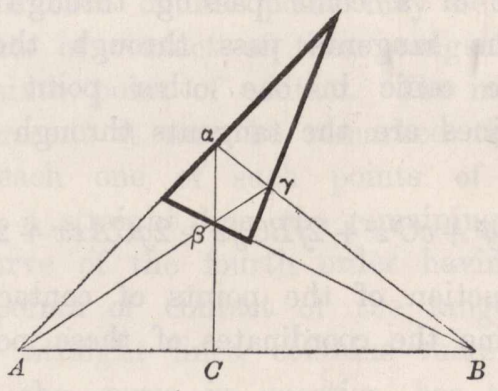

the lines joining the points of contact of the tangents from the double points. In fact, in the figure, if $\alpha \beta \gamma$ be the triangle whose sides are $\xi=0, \eta=0, \zeta=0$, and $A, B, C$ the points of intersection of the sides of this triangle with the line

$$
\frac{\xi}{F}+\frac{\eta}{G}+\frac{\zeta}{H}=0
$$


the lines in question are $A \alpha, B \beta, C \gamma$. Moreover, the points of contact upon $A \alpha$ are harmonics with respect to $A, \alpha$, and in like manner the points of contact on $B \beta, C \gamma$ are respectively harmonics with respect to $B, \beta$ and to $C, \gamma$. This proves that if three of the points of contact are in the same straight line, the remaining three are also in the same straight line; in fact, we may consider three of the points of contact as given by

$$
\begin{aligned}
& \xi: \eta: \zeta=\sqrt{ }(-a K): \quad G:-H, \\
& \xi: \eta: \zeta=-F: \sqrt{ }(-b K): H \text {, } \\
& \xi: \eta: \zeta=F:-G: \sqrt{ }(-c K) \text {; }
\end{aligned}
$$

and the condition that these may be in the same line is

$$
\sqrt{ }(-a) \sqrt{ }(-b) \sqrt{ }(-c)+G H \sqrt{ }(-a)+H F \sqrt{ }(-b)+F G \sqrt{ }(-c)=0,
$$

which remains unaltered when the signs of all the roots are changed. The equation just obtained may be considered as the condition which must exist between the coefficients of

$$
\frac{a}{x^{2}}+\frac{b}{y^{2}}+\frac{c}{z^{2}}+\frac{2 f}{y z}+\frac{2 g}{z x}+\frac{2 h}{x y}=0,
$$

in order that this curve may be transformable into the form

$$
\sqrt{ }\{\boldsymbol{\alpha} u(v+w-u)\}+\sqrt{ }\{\boldsymbol{\beta} v(w+u-v)\}+\sqrt{ }\{\boldsymbol{\gamma} w(u+v-w)\}=0 .
$$

